\title{
The Level of Syndecan-1 Expression is a Distinguishing Feature in Behavior between Keratoacanthoma and Invasive Cutaneous Squamous Cell Carcinoma
}

\author{
Perkins Mukunyadzi, M.D., Ralph D. Sanderson, Ph.D., Chun-Yang Fan, M.D., Ph.D., \\ Bruce R. Smoller, M.D. \\ Departments of Pathology (PM, RDS, CYF, BS) and Dermatology (BRS), University of Arkansas for Medical \\ Sciences and Central Arkansas Veterans Healthcare System, Little Rock, Arkansas
}

\begin{abstract}
Keratoacanthoma (KA) resolves spontaneously but is regarded by some as a variant of squamous cell carcinoma (SCC). However, others consider KA a totally benign entity. Syndecan- 1 is one of the heparan sulfate proteoglycans that mediates intercellular and cell to matrix adhesion. Its expression appears to be inversely correlated with tumor aggressiveness and invasiveness. Previous studies have shown decreased levels of syndecan-1 expression in invasive cutaneous SCC, correlating with tumor de-differentiation. However, a similar study has never been done on KA. To investigate syndecan-1 expression in classic KA and compare the results with those of classic invasive SCC, 24 KAs were immunostained for syndecan-1 (CD 138) using the monoclonal antibody B-B4 on formalin-fixed paraffin-embedded tissue. Results were semiquantitatively scored as either negative or positive (mild, moderate, or strong) and compared with those previously obtained on 23 invasive SCC and in situ lesions. All 24 KAs were positive for syndecan-1 expression. Staining intensity of 18 cases was comparable with that of SCC in situ or adjacent normal epidermis. By comparison, invasive SCC showed significantly diminished staining. Reduced staining in focal areas of cytologic atypia at the base was present in three KAs. Syndecan-1 expression in KA mirrors that of SCC in situ and normal epidermis, providing a molecular basis that biologically KA may be closely related to SCC in situ but distinctively different from invasive SCC.
\end{abstract}

Copyright (C) 2002 by The United States and Canadian Academy of Pathology, Inc.

VOL. 15, NO. 1, P. 45, 2002 Printed in the U.S.A.

Date of acceptance: October 9, 2001.

Presented in part at the 89th Annual Meeting of the US and Canadian Academy of Pathology, March 25-31, 2000, New Orleans, Louisianna. Published in abstract form in Modern Pathology 2000;13:66A.

Ralph D. Sanderson is supported by the National Institutes of Health, grant CA68494.

Address reprint requests to: Perkins Mukunyadzi, M.D., Central Arkansas Veterans Healthcare System, 4300W 7th Street, Little Rock, Arkansas 72205; e-mail: mukunyadziperkins@uams.edu; fax: 501-257-6430.
KEY WORDS: Behavior, Keratoacanthoma, Squamous cell carcinoma, Squamous cell carcinoma in situ, Syndecan-1.

Mod Pathol 2002;15(1):45-49

Keratoacanthomas (KAs) are skin epidermal tumors originating from the squamous epithelium. Although KA is known to spontaneously regress and disappear without specific therapy, most are removed by excision because of fear of possible malignant behavior. Their true natural biologic behavior thus remains unclear and controversial. Some regard KAs as benign, because of their tendency to resolve, whereas others consider them a variant of squamous cell carcinoma (SCC) (1-3). The microscopic evaluation of KA can be problematic, particularly at the base of the lesion, where distinguishing KA from invasive SCC may be difficult, if not impossible.

Syndecans are heparan sulfate cell surface proteoglycans that function in binding growth factors and other soluble molecules, and participate in cell-to-cell and cell to extracellular matrix (ECM) interactions $(4,5)$. In cellular interactions, syndecans are important in cell adhesion and cohesion, thus maintaining normal cell architecture, differentiation, growth, and migration. There are four members within the syndecan family, each having an extracellular, a transmembrane, and cytoplasmic domain. The extracellular domain contains covalently bound heparan sulfate chains that can bind several ECM molecules such as collagen, fibronectin, thrombospondin, and others (5). Syndecan-1, the most studied member of this family of cell surface proteoglycans is abundantly expressed in the normal stratified squamous epithelium (6-8). Importantly, syndecan-1 has been shown to inhibit invasion of cells into type 1 collagen gels $(9,10)$. Thus, loss of the syndecan-1 expression may be an important step in progression of carcinoma cells to the metastatic phenotype. In 
SCC of the head and neck, and larynx, the level of syndecan-1 expression is markedly reduced, correlating inversely with tumor aggressiveness (11-13). Our recent studies on syndecan-1 expression in cutaneous SCC showed a similar relationship, with the levels of expression significantly reduced in invasive SCC, but not in SCC in situ (14).

To explore the biologic differences between KA and cutaneous SCC, we investigated syndecan-1 expression in KA and compared the results with our previous findings of syndecan- 1 expression in $\mathrm{cu}$ taneous SCC and SCC in situ.

\section{MATERIALS AND METHODS}

The Pathology files at the University of Arkansas for Medical Sciences were searched for cases of KA. Twenty-four cases were selected from a total of 30 KAs diagnosed over a 2-year period (1997-1998). Of those not selected, five were outside consultations for which both the glass slides and paraffin blocks were not available, and one case had no suitable tissue left. All the cases were diagnosed as KA. The diagnosis of KA was based on the following histologic features: a symmetric crateriform tumor with orthokeratotic, parakeratotic cornified cells, central keratin plugging, undermining of the dermis beneath by the tumor, collarettes of proliferating keratinocytes at the edges, well differentiated squamous epithelium with eosinophilic or glassy cytoplasm, and atypia of peripheral keratinocytes. Regressing KA were characterized, in addition to the above features, by diminished proliferation of the epithelium with flattening of the floor of the tumor. The specimens were fixed in $10 \%$ formalin and embedded in paraffin. Sections $4 \mu \mathrm{m}$ in thickness were cut and mounted on silane-coated slides, air-dried and deparaffinized before antigen retrieval by the steam heat method for 40 minutes using the DAKO Target Retrieval solution (DAKO, Carpenteria, CA). The endogenous peroxidase activity was quenched with $0.3 \%$ hydrogen peroxide for 10 minutes and nonspecific binding was blocked by goat serum for 30 minutes. The DAKO Large Volume LSAB2 Alkaline Phosphatase Kit (DAKO) was used with a 1:100 dilution of the B-B4 primary antibody (Serotec, Raleigh NC), a mouse anti-human antibody that recognizes an epitope found in human syndecan-1 (CD 138) (15). Sections were incubated with primary antibody for $80 \mathrm{~min}$ utes at room temperature, followed by the biotinylated secondary antibody for 30 minutes and streptavidin alkaline phosphatase for 30 minutes. 3,3'-Diaminobenzidine chromogen was used to visualize the end product. Between each step, sections were washed with phosphate buffered saline, except for the last step before counter-staining where de-ionized water was used. Normal skin with strong syndecan-1 immunoreactivity in the keratinocytes was used as the positive control, and the negative control consisted of a 1:100 dilution of a nonspecific isotype-matched IgG1 mouse antibody, MCA 928 (Serotec) that was substituted for the B-B4 antibody. The slides were then counter stained with Hematoxylin before dehydration and cover slipping.

The same method and conditions, with comparable controls were applied throughout the procedure as those used in our previous study of 23 cutaneous SCC (acantholytic and non-acantholytic) and SCC in situ (14).

\section{RESULTS}

\section{Pathologic Findings}

Twenty of 24 (83\%) cases examined showed the typical microscopic appearance of KA (Fig. 1A): a crateriform architecture, central keratin plug, and lateral collarettes of proliferating squamous cells. There were no tumors with frank invasive disease but three KAs demonstrated focal areas of cytologic atypia (Fig. 2A). Five (21\%) cases were in different
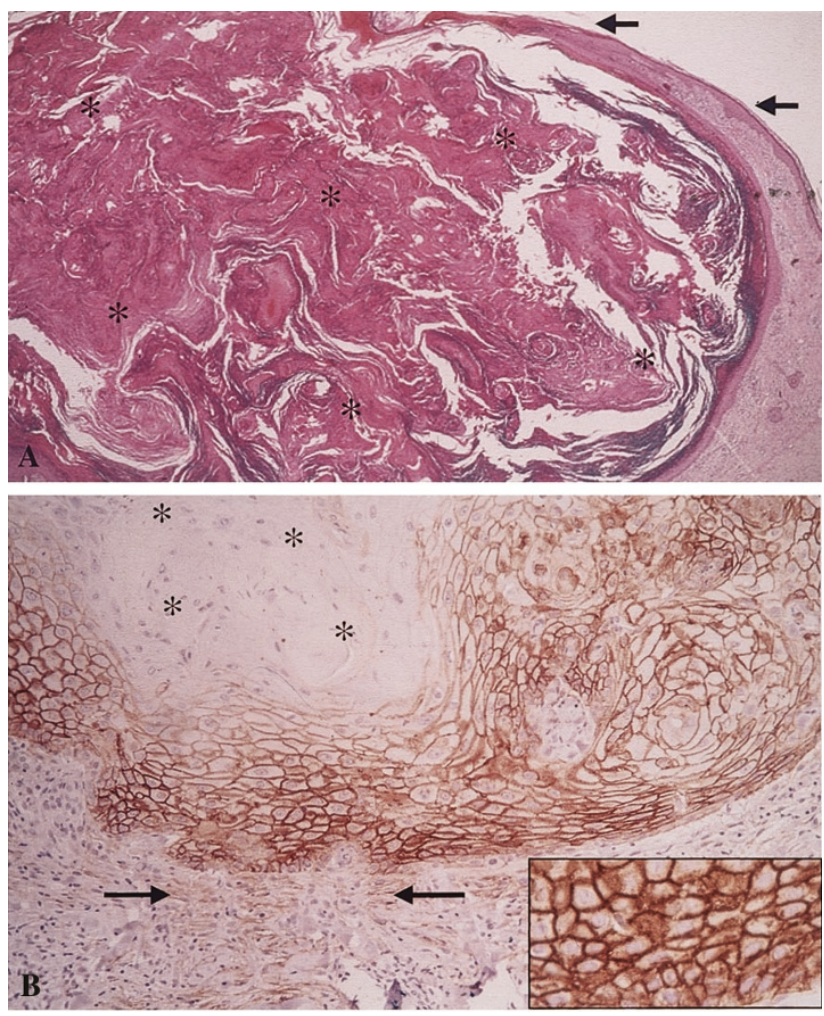

FIGURE 1. A, Classical keratoacanthoma with a keratin filled central crater (asterisk) and lateral buttresses (arrows) of proliferating squamous epithelium. B, Typical keratoacanthoma showing strong immunostaining for syndecan-1 on the squamous epithelium cell surface. Note weaker syndecan-1 expression is also detected in the underlying stroma (arrow). Insert, higher magnification of squamous epithelium (1B). 
stages of regression. A strong dermal lymphocytic response was noted in six KAs, whereas moderate and mild responses were present in six and nine cases, respectively (not shown). Three cases lacked any dermal lymphocytic response at all.

\section{Syndecan-1 Immunoreactivity}

Syndecan-1 immunoreactivity was semi-quantitatively analyzed and scored as follows: $(-)$ negative, $(+)$ mild, $(++)$ moderate, and $(+++)$ strong staining. All 24 KAs were positive for syndecan-1 expression, with $18(75 \%)$ cases showing strong staining (Fig. 1B), and six (25\%) demonstrating moderate staining (Table 1). Three (12.5\%) KAs with overall strong staining for syndecan-1, showed reduced levels of syndecan-1 expression (mild staining) in the focal areas of cytologic atypia (Fig. 2B). The level of staining intensity of the 18 cases with strong syndecan-1 expression (Fig. 1B) was comparable with that seen previously in the normal epidermis or SCC in situ (14). Five (21\%) cases of regressing KA showed strong epidermal expression of syndecan-1, and also demonstrated stromal staining in the dermis, subjacent to the KA (Fig. 3), a pattern not described in SCC (14). Staining was absent in the keratin plug of the KA crater and decreased in the parakeratotic cells adjacent to the keratin plug. Diminished staining for syndecan-1 in SCC is shown in Fig. 4.

The results show distinct differences in the level of syndecan-1 expression when compared with our previous findings in conventional cutaneous SCC (14), where the levels of syndecan-1 expression were markedly reduced in both invasive (Fig. 4) (four cases) and acantholytic (11 cases) SCC (not shown). Additionally, stromal syndecan-1 staining was noted in some regressing KA. However, there were no differences in the staining pattern and intensity when compared with SCC in situ (eight cases) and adjacent normal epidermis.

\section{DISCUSSION}

We examined the levels of syndecan-1 expression by immunohistochemistry in KA and compared the results with our previously published findings of syndecan-1 expression in cutaneous SCC and SCC in situ (14). Results show that the level of

TABLE 1. Level of Syndecan-1 Expression in 24 Keratoacanthomas (KA)

\begin{tabular}{|c|c|c|c|c|}
\hline & $\begin{array}{c}\text { Negative } \\
\text { Staining } \\
(-)\end{array}$ & $\begin{array}{c}\text { Mild } \\
\text { Staining } \\
(1+)\end{array}$ & $\begin{array}{c}\text { Moderate } \\
\text { Staining } \\
(2+)\end{array}$ & $\begin{array}{c}\text { Strong } \\
\text { Staining } \\
(3+)\end{array}$ \\
\hline $\begin{array}{l}\text { Number } \\
\text { of cases }\end{array}$ & 0 & 0 & $6^{*}$ & $18^{+}$ \\
\hline
\end{tabular}
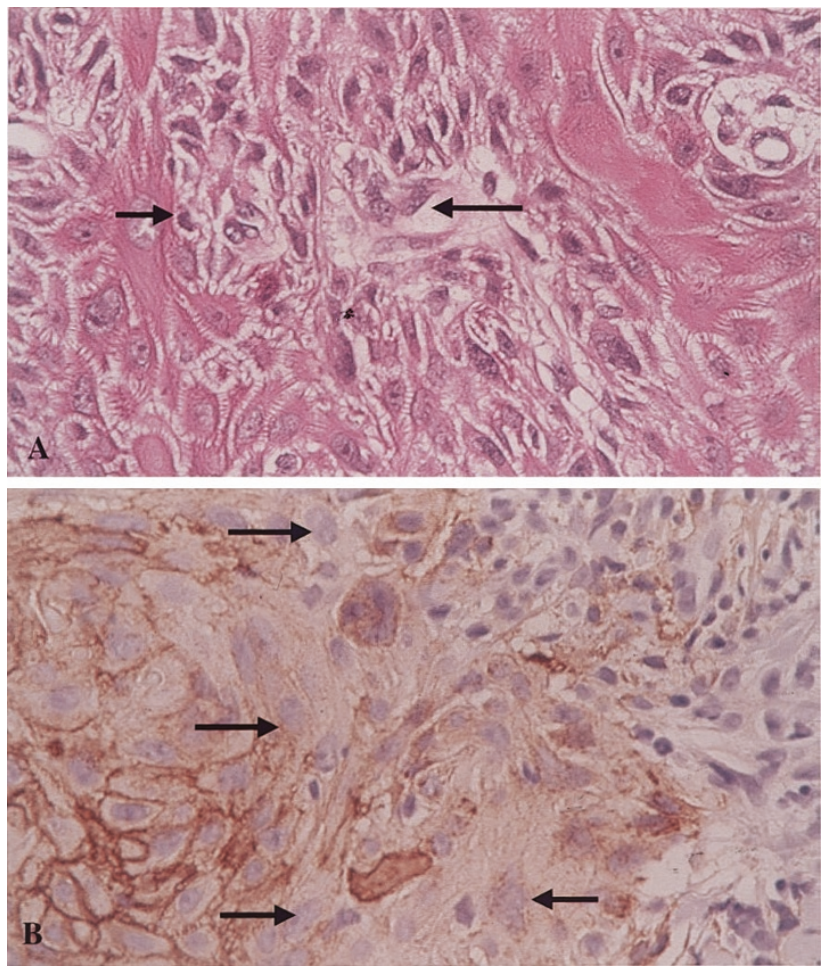

FIGURE 2. A, Cytologic atypia (arrows) in a keratoacanthoma. B, Atypical areas show diminished syndecan-1 expression (arrows) as compared with adjacent epithelium.

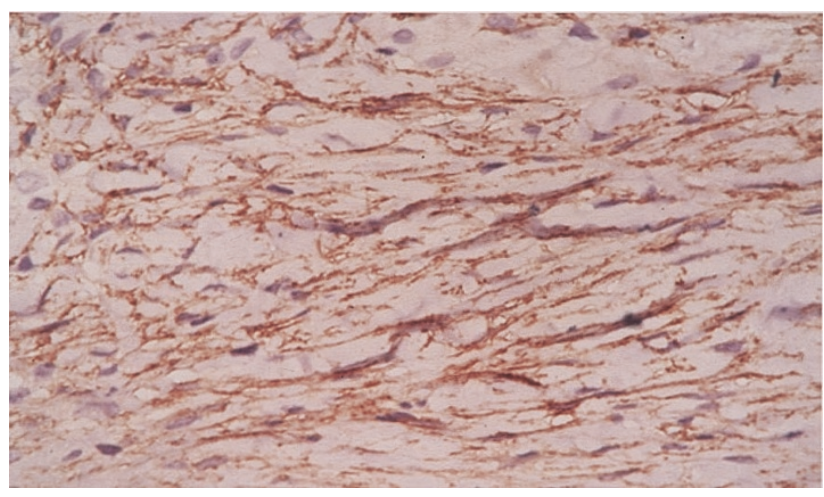

FIGURE 3. Stroma underlying a regressing keratoacanthoma show strong expression of syndecan-1.

syndecan-1 expression in KA is similar to that of SCC in situ, but much stronger than that of invasive SCC. The level of syndecan-1 expression in SCC in situ is the same as that seen in normal skin, where all keratinocytes show strong basolateral membrane staining $(16,17)$. These results suggest that immunophenotypically KA is more closely related to SCC in situ than to invasive SCC. We hypothesize that the biologic behavior of KA may be distinctly different from invasive SCC because significant reduction in syndecan-1 expression occurs only in invasive malignancies $(12,13)$.

Three $(12.5 \%)$ percent of the KA cases demonstrated diminished syndecan-1 expression in areas 


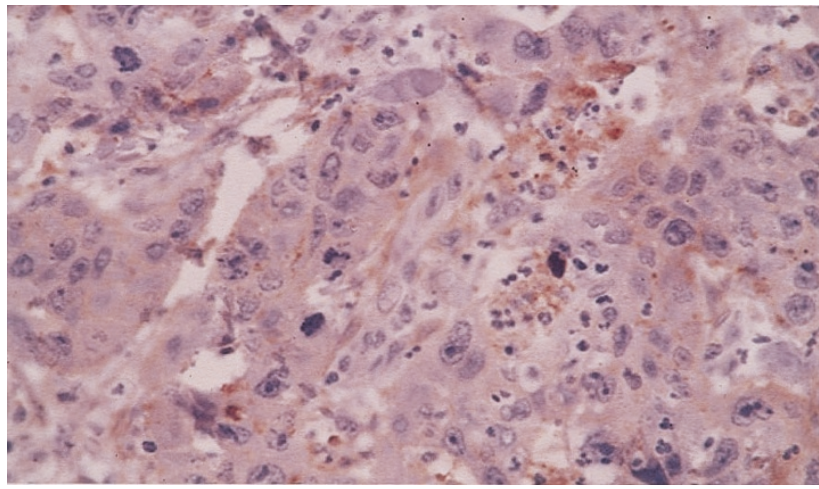

FIGURE 4. Invasive cutaneous squamous cell carcinoma showing reduced syndecan-1 immunostaining.

of cytologic atypia. Staining in these areas was mild to moderate. It has been suggested that syndecan-1, together with other proteoglycans is associated with cellular differentiation, cell-to-cell, and cell to ECM interactions. The level of syndecan-1 expression is maximal in normal epithelia, particularly in the stratified squamous epithelium of the skin (18), but shows gradual reduction with malignant transformation of the tumor cells $(14,19)$. Absence or diminished levels of syndecan-1 expression are seen in invasive SCC, correlating with the loss of cellular cohesion and the development of invasive and aggressive features (14).

In situ tumors express syndecan-1. It appears that the beginning of loss of syndecan-1 expression marks the onset of malignant transformation within the cells and that marked reduction in expression is an important indicator of an infiltrative or invasive growth pattern and cytologic atypia $(10,17)$. Although the expression of syndecan-1 in KA may not resolve the question of whether KA is neoplastic or not, the behavioral difference between KA and SCC appears related to their different levels of syndecan-1 expression. In cutaneous SCC with established invasiveness (including acantholytic type), marked reduction in syndecan-1 expression is present and the reduction is more apparent in areas of poor cellular differentiation. In head and neck SCC, P. Inki et al. demonstrated that intermediate or strong syndecan-1 expression was associated with smaller and well-differentiated tumors, whereas tumors with higher histologic grade had lower levels of syndecan-1 expression (13). Pulkkinen and colleagues obtained similar results in laryngeal carcinomas where patients with reduced levels of syndecan-1 expression had poorer prognosis (12).

Although KA and cutaneous SCC rarely result in patient death, morbidity and recurrences of the latter may be significant. There has always been controversy in their biologic relationship. Because of the potential of most KAs to regress spontaneously, perhaps due to a strong immune response, KAs are considered totally benign by some. However, because there are also tumors that show typical features of KA and yet demonstrate cytologic atypia and invasive features posing diagnostic challenges in distinguishing them from true SCC, others argue that KAs are a form of SCC. It could be argued that these two lesions represent different stages of the same disease, but it cannot be denied that as a group, KAs show unique clinico-pathologic features.

Five $(21 \%)$ cases of regressing KA demonstrated stromal expression of syndecan-1. The significance of this stromal induction of syndecan-1 expression and its relationship with the immune response (lymphocytic reaction) and KA regression is unclear and remains to be determined. Stromal syndecan-1 staining has been seen in some cases of invasive breast cancer (20), and we have also observed similar findings in some cases of malignant salivary gland tumors (unpublished data).

Because syndecan-1 acts as an inhibitor of cell invasion, retention of syndecan-1 expression in KA may account for its noninvasive behavior. This is in contrast to SCC, where syndecan-1 expression is lost and the tumor cells become invasive. Overall, our findings suggest that biologically, KAs are more closely related to SCC in situ of the skin than to invasive SCC.

\section{REFERENCES}

1. Beham A, Regauer S, Soyer H, Beham-Schmid C. Keratoacanthoma: a clinically distinct variant of well differentiated squamous cell carcinoma. Adv Anat Pathol 1998;5:269-80.

2. Leboit P. Is keratoacanthoma a variant of squamous cell carcinoma. New insights into an old controversy. . .soon? Am J Dermatopathol 1995;17:319-20.

3. Hodak E, Jones R, Ackerman A. Solitary keratoacanthoma is a squamous-cell carcinoma: three examples with metastases [see comments]. Am J Dermatopathol 1993;15:332-52.

4. Carey D. Syndecans: multifunctional cell-surface coreceptors. Biochem J 1997;327:1-16.

5. Bernfield M, Gotte M, Park P, Reizes O, Fitzgerald M, Lincecum J, et al. Functions of cell surface heparan sulfate proteoglycans. Annu Rev Biochem 1999;68:729-77.

6. Inki P, Larjava H, Haapasalmi K, Miettinen H, Grenman R, Jalkanen M. Expression of syndecan-1 is induced by differentiation and suppressed by malignant transformation of human keratinocytes. Eur J Cell Biol 1994;63:43-51.

7. Inki P, Gomez M, Quintanilla M, Cano A, Jalkanen M. Expression of syndecan in transformed mouse keratinocytes. Lab Invest 1992;62:225-33.

8. Sanderson R, Bernfield M. Molecular polymorphism of a cell surface proteoglycan: distinct structures on simple and stratified epithelia. Proc Natl Acad Sci USA 1988;85:9562-6.

9. Liebersbach B, Sanderson R. Expression of syndecan-1 inhibits cell invasion into type 1 collagen. J Biol Chem 1994; 269:20013-9.

10. Kato S, Saunders S, Nguyen H, Bernfield M. Loss of cell surface syndecan-1 causes epithelia to transform into anchorage-independent mesenchyme-like cells. Mol Biol Cell 1995;6:559-76. 
11. Anttonen A, Kajanti M, Heikkila P, Jalkanen M, Joensuu H. Syndecan-1 expression has prognostic significance in head and neck carcinoma. Br J Cancer 1999;79:558-64.

12. Pulkkinen J, Pentitinen M, Jalkanen M, Klemi P, Grenman R. A new prognostic marker in laryngeal cancer. Acta Otolaryngol (Stockh) 1997;117:312-5.

13. Inki P, Joensuu H, Grenman R, Klemi P, Jalkanen M. Association between syndecan-1 expression and clinical outcome in squamous cell carcinoma of the head and neck. Br J Cancer 1994;70:319-23.

14. Bayer-Garner I, Sanderson R, Smoller B. Syndecan-1 expression is diminished in acantholytic cutaneous squamous cell carcinoma. J Cutan Pathol 1999;26:386-90.

15. Wijdenes J, Vooijs W, Clement C, Post J, Morard F, Vita N, et al. A plasmocyte selective monoclonal antibody (B-B4) recognizes syndecan-1. Br J Haematol 1996;94:18-23.
16. Inki P, Stenback F, Grenman S, Jalkanen M. Immunohistochemical localization of syndecan-1 in normal and pathological human uterine cervix. J Pathol 1994;172:349-55.

17. Inki P, Stenback F, Tavel L, Jalkanen M. Immunolocalization of syndecan in mouse skin after skin tumors induced by UV irradiation. Loss of expression associated with malignant transformation. Am J Pathol 1991;139:1333-40.

18. Sanderson R, Hinkes M, Bernfield M. Syndecan-1, a cellsurface proteoglycan, changes in size and abundance when keratinocytes stratify. J Invest Dermatol 1992;99: $390-6$.

19. Inki P, Jalkanen M. The role of syndecan-1 in malignancies. Annu Med 1996;28:63-7.

20. Stanley M, Stanley M, Sanderson R, Zera R. Syndecan-1 expression is induced in the stroma of infiltrating breast carcinoma. Am J Clin Pathol 1999;112:377-83. 